\title{
On Fractionally-Spaced Equalizer Design for Digital Microwave Radio Channels
}

\author{
C. R. Johnson, Jr., H. J. Lee, J. P. LeBlanc, T. J. Endres, R. A. Casas, E. Tai, Z. Reznic, \\ W. E. Meyer, F. López de Victoria, J. R. Treichler, I. Fijalkow, and Z. Ding
}

\begin{abstract}
Recent advances in blind identification of fractionally-spaced models for digital communication channels and blind fractionally-spaced equalizer adaptation rely on the assumption that the time span chosen for the fractionally-spaced equalizer exceeds that of the channel. This paper considers time-domain design formulas minimizing the mean-squared symbol recovery error achieved by a finite-length FIR fractionally-spaced equalizer with a time span shorter than the channel impulse response time span for white zero-mean $Q \Lambda \mathrm{M}$ sources in the presence of white zero-mean channel noise. For minimum mean-squared error designs the symbol error rates achievable are plotted versus the ratio of the source variance to the channel noise variance (with the channel model power normalized to achieve a received signal of unit variance) for different fractionally-spaced equalizer lengths on 64-QAM for several $T / 2$-spaced channel models derived from experimental data. Our intent is to fuel the ongoing debate about fractionally-spaced equalizer length selection.
\end{abstract}

\section{Introduction}

This paper considers the design of a fractionallyspaced equalizer from a data-based fractionally-spaced channel model. Refer to Figure 1. $T$ is the time between source symbols. In the absence of channel noise $n$, a $T / 2$-spaced channel model converts the $T / 2$-spaced input sequence (which is composed from the $T$-spaced source symbol sequence $s$ interleaved with zeros at the fractional symbol intervals) into the twice per symbol samples of the received signal $r$ at the equalizer input. For simplicity, in this image all samplers are assumed to be synchronized with the symbol times.

In [1], $T / 2$-spaced channel models with over 200 "active" consecutive taps are derived for digital microwave radio channels from experimentally acquired received signal streams. (Though [1] is not available publicly, we anticipate that a portion of the database acquired and used in [1] will soon be made available through the National Science Foundation's Signal Processing Information Base (SPIB) administered at Rice University.) In our designs, as often occurs in this digital microwave radio context, the $T / 2$-spaced equalizer tapped-delay-line length will have no more taps than the associated $T / 2$-spaced channel model. (The related minimum mean-squared error design problem when the fractionally-spaced equalizer time span exceeds that of the channel is considered in [2].)

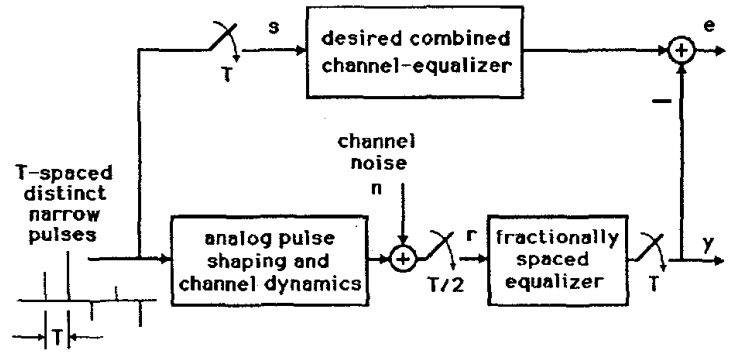

Figure 1: Linear Fractionally-Spaced Equalization

A number of exciting results have been reported, e.g. [3]-[9], in a recent swell in interest in the blind identification and equalization of communication channels. Blind identification/equalization is performed from just the received signal record without access to the associated source symbol sequence. One of the key assumptions in this work is that the equalizer time span need not exceed that of the channel in the noise-free case for perfect equalization. In microwave radio channel applications, e.g. the ones cited in this paper, this length requirement can prove too costly - and unnecessary.

In the presence of channel noise, an appealing (and pragmatic) performance measure, with both source symbol sequence $s$ and fractionally-sampled channel noise sequence $n$ white and zero mean and each uncorrelated with the other, is the variance of the symbol

Johnson, Lee, LeBlanc, Endres, Casas, Reznic, López de Victoria, Meyer, and Tai are (or were) with the School of Electrical Engineering, Cornell University, Ithaca, NY 14853 USA. Treichler is with Applied Signal Technology, Sunnyvale, CA. Fijalkow is with Groupe ETIS, ENSEA de Cergy, Cergy-Pontoise, France. Ding is with the Department of Electrical and Computer Engineering, Auburn University, Auburn, AL. The authors list includes all members of the Cornell University Blind Equalization Research Group and collaborative visitors in the months prior to ICASSP95 when discussion of these "real" problems was rampant. 
recovery error [10]. The symbol recovery error $e$ is the difference between the downsampled equalizer output $y$ and the source symbol sequence $s$ passed through the $T$-spaced dynamics desired of the combined channelequalizer response between $s$ and $y$, typically a delay. Inclusion of channel noise effects in the symbol recovery error variance results in regularization of the Sylvester matrix that would otherwise be (pseudo)inverted in solving the Diophantine equation of the noise-free case. (Peek ahead to (8).)

Though the design equations used here are for minimizing mean squared symbol recovery error, symbol error rate (SER) is a performance measure more closely related to practical performance concerns. From [11], the probability of an incorrect decision or symbol error for an $M$-ary QAM system is

$$
S E R=1-\left[1-\left(1-\frac{1}{\sqrt{M}}\right) \operatorname{erfc}\left(\sqrt{\frac{3 \sigma_{s}^{2}}{2(M-1) \sigma_{e}^{2}}}\right)\right]^{2}
$$

where $\sigma_{s}^{2}$ is the variance of the white, zero-mean source and $\sigma_{e}^{2}$ is the mean squared symbol recovery error achieved by the equalizer. The ratio $\sigma_{s}^{2} / \sigma_{e}^{2}$ is the SNR of the recovered signal (i.e. the equalizer output). We will use (1) to calibrate our designs. A reason (beyond tractability) for considering an FSE design based on minimizing mean-squared error (MSE) rather than minimizing SER is that a number of adaptive algorithms attempt via an approximate gradient descent procedure to minimize MSE, such as decision-directed LMS which is the linear FSE blind adaptation algorithm of choice for convergent-tracking in numerous digital radio applications.

The following two sections (a) formulate the design equation for the equalizer providing the minimum mean squared recovery error given knowledge of the source to noise variance ratio but fewer equalizer taps than needed to produce zero symbol recovery error with channel noise absent and (b) briefly describe the experiments used to generate the data-based channel models under study and examine achievable performance in equalizing these models in terms of the SER for various equalizer lengths and a 64-QAM source.

\section{Minimum MSE FSE Design}

Consider the following communications system problem. The source sequence is a set of complex values drawn from a QAM constellation. This source sequence can be viewed as triggering transmission of an analog signal through an analog medium with the received signal sampled every $T / 2$ seconds. The $T / 2$-spaced equalizer is a causal, finite-impulse-response, tapped-delayline that filters the $T / 2$-sampled received signal with potentially nonzero coefficients $f_{i}$ for $i=0$ to $N$ and a unit delay of $T / 2$, i.e. half the symbol period.

The relationship between the $z$-transform of the source sequence $S(z)$ and the downsampled version (i.e. every other sample) of the equalizer output $Y(z)$ is a multichannel model discussed in [3]-[9]. For an interpretation of the result of this multichannel modelling, consider first a sampled-data model of the distortion created by the $T / 2$-spaced samples of the linear channel output for an arbitrary source with only 6 nonzero impulse response coefficients $c_{i}$ for $i=0$ to 5 . The objective is design of a fractionally-spaced FIR linear equalizer, which is a weighted, tapped-delay-line with a unit delay of $T / 2$ seconds and four weights $f_{i}$ for $i=0$ to 3 . The combined channel-equalizer $T / 2$-sampled impulse response has 9 coefficients $h_{i}$ for $i=0$ to 8 . These coefficients are related by

$$
\left[\begin{array}{l}
h_{0} \\
h_{1} \\
h_{2} \\
h_{3} \\
h_{4} \\
h_{5} \\
h_{6} \\
h_{7} \\
h_{8}
\end{array}\right]=\left[\begin{array}{llll}
c_{0} & 0 & 0 & 0 \\
c_{1} & c_{0} & 0 & 0 \\
c_{2} & c_{1} & c_{0} & 0 \\
c_{3} & c_{2} & c_{1} & c_{0} \\
c_{4} & c_{3} & c_{2} & c_{1} \\
c_{5} & c_{4} & c_{3} & c_{2} \\
0 & c_{5} & c_{4} & c_{3} \\
0 & 0 & c_{5} & c_{4} \\
0 & 0 & 0 & c_{5}
\end{array}\right]\left[\begin{array}{l}
f_{0} \\
f_{1} \\
f_{2} \\
f_{3}
\end{array}\right] .
$$

The effect of fractional spacing, multichannel modelling, and downsampling is the same as removing every other row from (2). This paradigm can be supported by accepting the source sequence as nonzero only every other $T / 2$-spaced sample, such that the downsampled version of the equalizer output will only consist of terms weighted by the combined channel-equalizer impulse response coefficients displaced an integer multiple of $T$ seconds from the sample time of the desired delay. For example, if a delay of $3 T / 2$ seconds represents the desired combined channel and downsampled equalizer response (i.e. $h_{3}=1$ and $h_{i}=0$ for $i \neq 3$ ), the multichannel model of fractionally-spaced equalization reduces (2) to the design equation

$$
\left[\begin{array}{l}
0 \\
1 \\
0 \\
0
\end{array}\right]=\left[\begin{array}{cccc}
c_{1} & c_{0} & 0 & 0 \\
c_{3} & c_{2} & c_{1} & c_{0} \\
c_{5} & c_{4} & c_{3} & c_{2} \\
0 & 0 & c_{5} & c_{4}
\end{array}\right]\left[\begin{array}{l}
f_{0} \\
f_{1} \\
f_{2} \\
f_{3}
\end{array}\right]
$$

which, with the appropriate definitions, can be written as $\mathbf{h}=\mathrm{Cf}$. $T$-spaced impulse responses other than a bulk delay from the source symbol to the downsampled equalizer output are also feasible objectives for the left side of (3).

What if the number of equalizer taps is insufficient for the design equation to have an exact solution? For 
example, imagine how (2) changes if only three consecutive taps are permitted to have nonzero values, i.e. $f_{i}$ nonzero only for $i=0,1$, and 2 or $i=1,2$, and 3 . With reference to (2), $f_{0}=0$ implies that the specific values in the first column of the channel impulse response matrix are inconsequential in satisfying (2), which could as well be reduced by eliminating this column. But, the first equation/row may as well also be removed because the remaining nonzero $f_{i}$ cannot influence $h_{0}$. For a midrange desired delay, the desired $h_{0}$ would be zero anyway. Thus, (2) becomes

$$
\left[\begin{array}{l}
h_{1} \\
h_{2} \\
h_{3} \\
h_{4} \\
h_{5} \\
h_{6} \\
h_{7} \\
h_{8}
\end{array}\right]=\left[\begin{array}{lll}
c_{0} & 0 & 0 \\
c_{1} & c_{0} & 0 \\
c_{2} & c_{1} & c_{0} \\
c_{3} & c_{2} & c_{1} \\
c_{4} & c_{3} & c_{2} \\
c_{5} & c_{4} & c_{3} \\
0 & c_{5} & c_{4} \\
0 & 0 & c_{5}
\end{array}\right]\left[\begin{array}{l}
f_{1} \\
f_{2} \\
f_{3}
\end{array}\right]
$$

$T / 2$-spaced equalizer design effectively "removes" either the four even rows or the four odd rows, leaving in both cases an equation of the form $\alpha=\beta \gamma$ where $\alpha$ is $4 \times 1$, $\beta$ is $4 \times 3$, and $\gamma$ is $3 \times 1$.

With reference to $(2)$, setting $f_{\mathbf{3}}=0$ (instead of $f_{0}$ ) removes the last column from the impulse response matrix filled with the $c_{i}$ in (2) resulting in an alternative to (4) with the matrix containing $c_{i}$ the same in both cases and the first index of 0 in $f$ matching that in $h$. Again there are two choices for removing every other row, both of which lead to a version of (4) where $h$ and $\mathbf{C}$ have more rows that $\mathrm{f}$. Thus, if the range of possible choices for the single nonzero tap in the desired $\mathbf{h}$ is limited to locations in (4) or the similar version with $f_{3}$ zeroed, they present only one matrix equation to row-decimate and solve rather than two as it seems. There will only be two choices for row decimation for a $T / 2$-spaced equalizer design. The "best" location for the delay in $\mathbf{h}$ (in an MSE sense) would then require resolution for the best design presuming the particular delay achieved is unimportant.

For both the source $s$ and the sampled channel noise $n$ white and zero-mean with variances $\sigma_{s}^{2}$ and $\sigma_{n}^{2}$ respectively, the symbol recovery error variance $\sigma_{e}^{2}$ is

$$
\sigma_{e}^{2}=(\mathbf{h}-\mathbf{C f})^{T}(\mathbf{h}-\mathbf{C f}) \sigma_{s}^{2}+\mathbf{f}^{T} \mathbf{f} \sigma_{n}^{2}
$$

where $\mathbf{h}$ represents the desired symbol-spaced combined channel-equalizer and $\mathbf{C f}$ is the achieved combination. The gradient (assuming real arithmetic) of the symbol recovery error variance $\sigma_{e}^{2}$ as a function of the equalizer parameter vector $f$ is

$$
\frac{\partial \sigma_{e}^{2}}{\partial \mathbf{f}}=\frac{\partial}{\partial \mathrm{f}}\left[\sigma_{s}^{2} \mathbf{f}^{T}\left(\mathbf{C}^{T} \mathbf{C f}+\mathbf{f} \frac{\sigma_{n}^{2}}{\sigma_{s}^{2}}-2 \mathbf{C}^{T} \mathbf{h}\right)\right]
$$

$$
=2 \sigma_{s}^{2}\left\{\left[\mathbf{C}^{T} \mathbf{C}+I_{N} \frac{\sigma_{n}^{2}}{\sigma_{s}^{2}}\right] \mathbf{f}-\mathbf{C}^{T} \mathbf{h}\right\}
$$

where $I_{N}$ is an $N \times N$ identity matrix. Using the regularized pseudo-inverse of $\mathrm{C}$ to compute $\mathbf{f}$ via

$$
\mathbf{f}=\left(\mathbf{C}^{T} \mathbf{C}+I_{N} \lambda\right)^{-1} \mathbf{C}^{T} \mathbf{h}
$$

zeros the gradient of (6) when $\lambda=\sigma_{n}^{2} / \sigma_{s}^{2}$, which is the inverse of the $s$ to $n$ SNR. For complex values in $C, h$, and $f, C^{T}$ is replaced by its complex conjugate transpose as in (83) in [3].

For $\mathrm{f}$ computed via $(7),(5)$ becomes

$$
\frac{\sigma_{e}^{2}}{\sigma_{s}^{2}}=\mathbf{h}^{T}\left[(I-\mathbf{C} M)^{T}(I-\mathbf{C} M)+\lambda M^{T} M\right] \mathbf{h}
$$

where $M=\left(\mathbf{C}^{T} \mathbf{C}+\lambda I_{N}\right)^{-1} \mathbf{C}^{T}$. For h a simple delay, $\mathbf{h}=\left[\begin{array}{llllllll}0 & \ldots & 0 & 1 & 0 & \ldots & 0\end{array}\right]^{T}$ where with the $d$ th element nonzero this represents a $(d-1) T$-second delay (plus $T / 2$ seconds if the even rows of the full $T / 2$ spaced channel impulse response matrix as in (2) have been removed instead of the odd ones). Note how $\mathbf{h}=\left[\begin{array}{llllll}0 & \ldots & 0 & 1 & 0 & \ldots\end{array}\right]^{T}$ simply extracts a particular column from $M$ in (7). The location of this delay could be considered a design variable. Various nonzero indices $d$ will result in the extraction of different diagonal elements of $\left[(I-\mathbf{C} M)^{T}(I-\mathbf{C} M)+\lambda M^{T} M\right]$ in (8). Thus, the index $d$ of the nonzero element in the optimum (i.e. minimum MSE) over all possible delays matches that of the smallest diagonal element of $\left[(I-\mathrm{C} M)^{T}(I-\mathrm{C} M)+\lambda M^{T} M\right]$ where $M=\left(\mathbf{C}^{T} \mathbf{C}+\lambda I_{N}\right)^{-1} \mathbf{C}^{T}$.

For comparisons across channels and SNRs, the received signal variance $\sigma_{r}^{2}=(1 / 2) \mathrm{c}^{T} \mathrm{c} \sigma_{s}^{2}+\sigma_{n}^{2}$ is always set to unity by a normalized $\mathrm{c}$, such that $\mathrm{c}^{T} \mathrm{c}=\frac{2\left(1-\sigma_{x}^{2}\right)}{\sigma_{s}^{2}}$ where $\mathbf{c}$ is the vector of channel impulse response coefficients $c_{i}$.

\section{Turning Experimental Received Signal Records into Equalizer Designs}

In the late 1980s Applied Signal Technology gathered direct measurements on long records of received QPSK and QAM sequences [1]. A $T / 2$-spaced blind equalizer using the constant modulus algorithm (CMA) [12] was applied to this data, followed (after eye opening) by decision-directed LMS until the recovered constellation was tightly clustered around the QAM source alphabet points in the complex plane. Using nearest element quantization produced an (essentially) errorfree version of the source sequence. This recovered source symbol sequence (interspersed with zeros) and the (mildly noisy) received signal now form an inputoutput pair that can be used to identify a $T / 2$-spaced 
model of the channel. This approach was taken to identify $T / 2$-spaced 200-plus tap, FIR channel models in numerous locations throughout the San Francisco Bay Area. These channels for which we have the identified $T / 2$-spaced complex channel impulse response were labelled "g201", "j201", "g401", "h401", and "g501".

The determination of the "optimum" (across all possible delays in $\mathbf{h}$ ) $\mathbf{f}$ minimizing the mean squared recovery error from the solution of (7) was performed for various SNRs (computed as $10 \log \left(\sigma_{s}^{2} / \sigma_{n}^{2}\right)$ ) and equalizer lengths of powers of 2. Figures 2-6 provide (a) the magnitudes of the complex impulse response and (b) the SER (for a 64-QAM source via (1)) versus SNR plots for various equalizer lengths. In each of the SER versus SNR $\left(\sigma_{s}^{2} / \sigma_{n}^{2}\right)$ plots in the parts (b), the solid line is the SER in the absence of channel dynamics when the channel is a single gain $c_{I}\left(=\sqrt{2\left(1-\sigma_{n}^{2}\right) / \sigma_{s}^{2}}\right)$ and the equalizer is a single gain $f_{I}\left(=c_{i} /\left(\lambda+c_{I}^{2}\right)\right)$. Being only $2 \mathrm{~dB}$ off this "ideal" curve for an SER of $10^{-6}$ is a typical performance objective.

With a specification of an SER of $10^{-6}$ and an SNR $2 \mathrm{~dB}$ off ideal for 64-QAM (i.e. $\sigma_{s}^{2} / \sigma_{n}^{2}=26.2 \mathrm{~dB}$ ) the following $T / 2$-spaced equalizer lengths in number of taps and desired delay in $T / 2$ second units result from solution of (7) and use of the delay optimization procedure associated with (8): (Channel/Desired Delay Index/FSE Length) g201/25/38; j201/24/59; g401/54/14; and g501/30/177. For channel h401, no equalizer shorter than the channel model proves satisfactory. However, in all other cases, the minimum adequate equalizer time span is considerably less than the time span of the channel impulse response. One might attempt to interpret the "minimum length" equalizer time span as matching that of the "significant" portion of the channel impulse responses in parts (a) of Figures 2-6. What features define "significant"?

\section{References}

[1] R. Gooch, B. Daellenbach, and E. Tsui, "Wideband PCM Channel Characterization Study: Final Report (Revision 2)," Applied Signal Technology Technical Report FR-020-87R2, May 1988.

[2] I. Fijalkow, A. Touzni, and C. R. Johnson, Jr., "Spatio-Temporal Equalizability under Channel Noise and Loss of Disparity," Proc. 15e Colloque GRETSI, Sept. 1995.

[3] L. Tong, G. Xu, and T. Kailtath, "Blind Identification and Equalization Based on Second Order Statistics: A Time Domain Approach," IEEE Trans. on Info. Thy., March 1994.

[4] E. Moulines, P. Duhamel, J-F. Cardoso, and S. Mayrargue, "Subspace Methods for the Blind Identification of Multichannel FIR Filters," IEEE Trans. on
Sig. Proc., Feb. 1995.

[5] D. Slock, "Blind Fractionally-Spaced Equalization, Perfect Reconstruction Filter Banks and Multichannel Linear Prediction," Proc. IEEE 1994 Int. Conf. on Acoustics, Speech, and Sig. Proc., April 1994.

[6] I. Fijalkow, F. López de Victoria, and C. R. Johnson, Jr., "Adaptive Fractionally Spaced Blind Equalization,". Proc. 6th IEEE DSP Workshop, October 1994.

[7] Y. Li and Z. Ding, "Global Convergence of Fractionally Spaced Godard Equalizers," Proc. 28th Asilomar Conf. on Signals, Systems, and Computers, Nov. 1994. [8] J. K. Tugnait, "On Blind Identifiability of Multipath Channels Using Fractional Sampling and SecondOrder Cyclostationary Statistics," IEEE Trans. on Info. Thy., Jan. 1995.

[9] L. Tong, G. Xu, B. Hassibi, and T. Kailath, "Blind Channel Identification Based on Second-Order Statistics: A Frequency-Domain Approach," IEEE Trans. on Info. Thy., January 1995.

[10] R. D. Gitlin, J. F. Hayes, and S. B. Weinstein, Data Communication Principles, Plenum, section 7.4, 1992.

[11] J. G. Proakis, Digital Communications, McGrawHill, 2nd ed., sect. 4.2.8, 1989.

[12] V. Wolff, R. Gooch, and J. R. Treichler, "Specification and Development of an Equalizer Demodulator for Wideband Digital Microwave Radio Signals," WESCON Conf. Record, 1987.

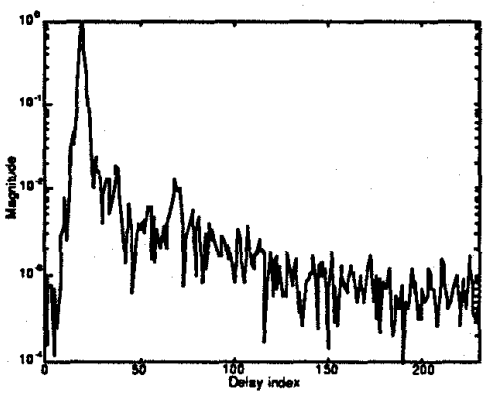

(a) impulse response

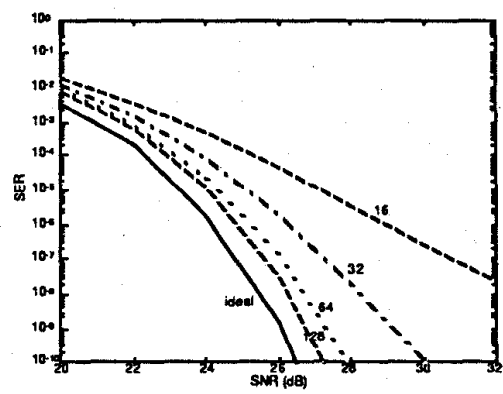

(b) symbol error rate Figure 2: Channel g201 


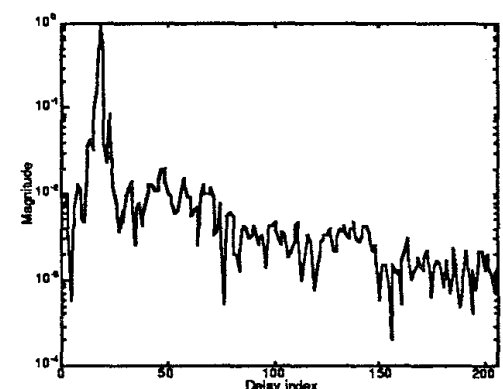

(a) impulse response

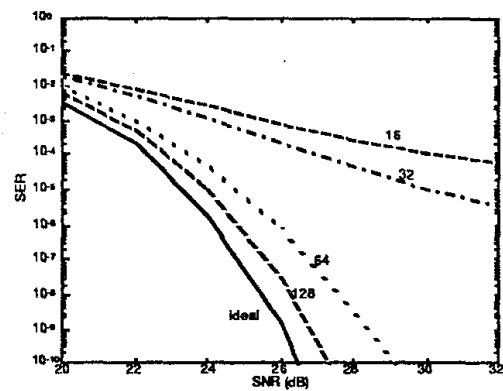

(b) symbol error rate

Figure 3: Channel j201

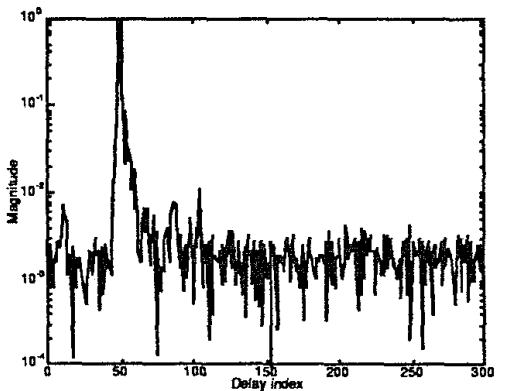

(a) impulse response

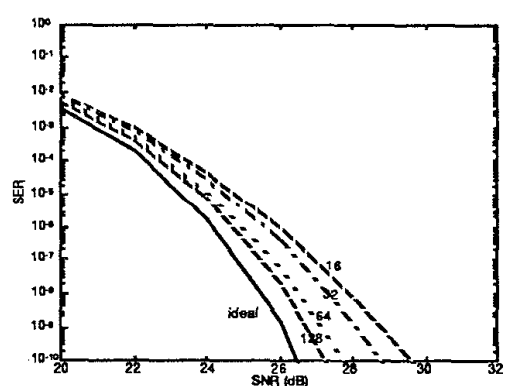

(b) symbol error rate Figure 4: Channel g401

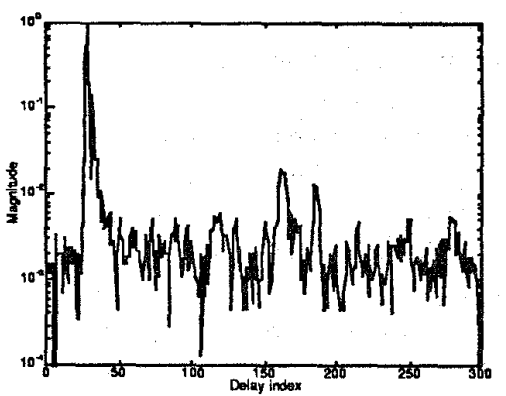

(a) impulse response

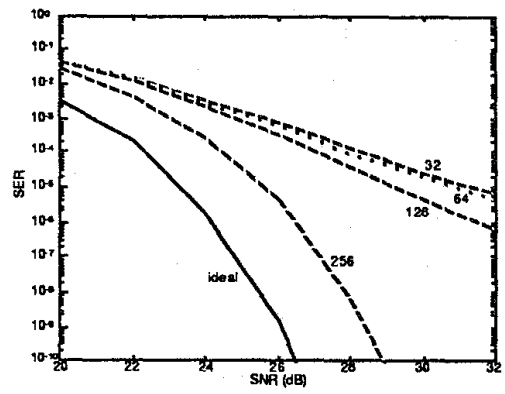

(b) symbol error rate

Figure 5: Channel h401

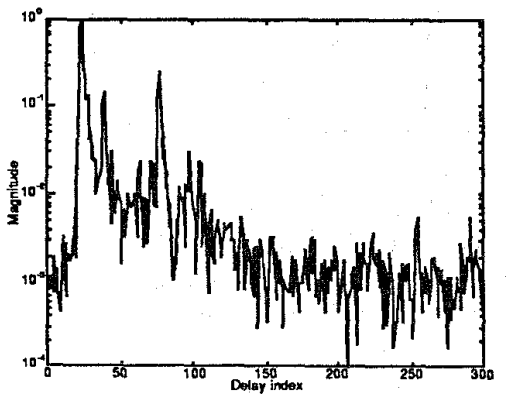

(a) impulse response

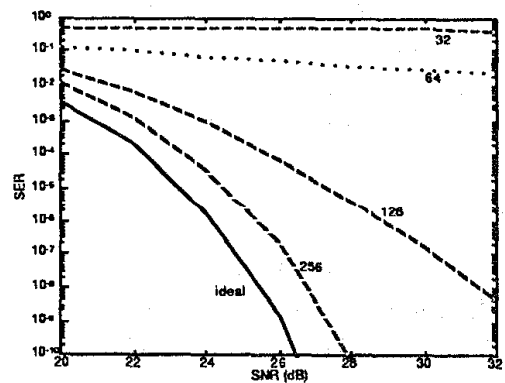

(b) symbol error rate Figure 6: Channel g501 